\title{
PTPRCAP is differentially expressed in lymph node metastasis in human breast cancer.
}

Shahan Mamoor, MS ${ }^{1}$

${ }^{1}$ shahanmamoor@gmail.com

East Islip, NY USA

Metastasis to the brain is a clinical problem in patients with breast cancer ${ }^{1-3}$. Between the breast and the brain reside the secondary lymphoid organ, the lymph nodes. We mined published microarray data ${ }^{4,5}$ to compare primary and metastatic tumor transcriptomes for the discovery of genes associated with lymph node metastasis in humans with metastatic breast cancer. We found that protein tyrosine phosphatase, receptor type $\mathrm{C}$ associated protein, encoded by PTPRCAP, was among the genes whose expression was most different in the lymph node metastases of patients with metastatic breast cancer as compared to primary tumors of the breast. Analysis of a separate microarray dataset revealed that PTPRCAP was also differentially expressed in brain metastatic tissues ${ }^{5}$. PTPRCAP mRNA was present at increased quantities in lymph node metastatic tissues as compared to primary tumors of the breast. Expression of PTPRCAP in primary tumors was correlated with patient distant metastasis-free survival in lymph node negative patients but not in lymph node positive patients. Modulation of PTPRCAP expression may be relevant to the biology by which tumor cells metastasize from the breast to the lymph nodes in humans with metastatic breast cancer.

Keywords: breast cancer, metastasis, lymph node metastases, central nervous system metastases, protein tyrosine phosphatase, receptor type $\mathrm{C}$ associated protein, PTPRCAP, systems biology of breast cancer, targeted therapeutics in breast cancer. 
One report described a 34\% incidence of central nervous system metastases in patients treated with trastuzumab for breast cancer ${ }^{2}$. More recently, the NEfERT-T clinical trial ${ }^{6}$ which compared administration of either neratinib or trastuzumab in conjunction with paclitaxel demonstrated that in a randomized, controlled setting, in breast cancer patients treated with neratinib, not only was the incidence of central nervous system recurrence significantly lower, the time to central nervous system metastasis was significantly delayed as compared to patients administered trastuzumab ${ }^{6}$. The alarmingly high rate of central nervous system metastasis described, as well as data, both anecdotal ${ }^{2}$ and from a randomized, controlled setting ${ }^{6}$ illustrating that treatment with trastuzumab may be associated with these events demands an enhanced understanding of the transcriptional makeup of lymph node metastatic tissues to support identification of therapeutic targets, whether they are treatment related or not. We performed a global comparative analysis of primary and metastatic tumors in patients with lymph node metastatic breast cancer ${ }^{4,5}$. We discovered differential and increased expression of the gene encoding protein tyrosine phosphatase, receptor type $\mathrm{C}$ associated protein, PTPRCAP, in lymph node metastatic tissues of patients with metastatic breast cancer.

\section{Methods}

We used datasets GSE $10893^{4}$ and GSE1259895 for this global differential gene expression analysis of lymph node metastatic breast cancer in conjunction with GEO2R. GSE10893 was generated using Agilent-011521 Human 1A Microarray G4110A technology with $n=11$ primary breast tumors and $n=3$ lymph node metastases from patients with breast cancer; analysis was performed using platform GPL 885 . GSE125989 was generated using Affymetrix Human Genome U133A 2.0 array technology with $n=16$ primary breast tumors and $n=16$ brain metastases from patients with breast cancer; the samples utilized by investigators for this microarray dataset were paired, matched tumor and metastasis samples; analysis was performed using platform GPL571. The Benjamini and Hochberg method of $p$-value adjustment was used for ranking of differential expression but raw $p$-values were used to assess statistical significance of global differential expression. Log-transformation of data was auto-detected, and the NCBI generated category of platform annotation was used. A statistical test was performed to evaluate whether PTPRCAP gene expression was significantly different between primary tumors of the breast and lymph node metastases in humans with breast cancer using a two-tailed t-test. For Kaplan-Meier survival analysis, we used the Kaplan-Meier plotter online tool ${ }^{7}$ for correlation of PTPRCAP mRNA expression levels with distant metastasis-free survival in $n=1309$ lymph node negative patients and $n=889$ lymph node positive patients.

\section{$\underline{\text { Results }}$}

We performed global comparative transcriptome analysis of metastatic tumor tissues of patients with metastatic breast cancer using published microarray data ${ }^{4,5}$ to describe the transcriptional landscape of lymph node metastasis in human breast cancer in an unbiased fashion and for the discovery of novel therapeutic targets.

\section{PTPRCAP is differentially expressed in the lymph node metastases of patients with lymph node} metastatic breast cancer.

Through blind, systems-level analysis of published microarray data ${ }^{4}$, we identified protein tyrosine phosphatase, receptor type $\mathrm{C}$ associated protein, encoded by PTPRCAP, as a differentially expressed gene in the breast metastatic tissues of humans with breast cancer (Table 1). When sorting each of the genes expressed in lymph node metastases based on significance of difference as compared to primary tumors of the breast in patients with breast cancer, PTPRCAP ranked 155 out of 18034 total transcripts (Chart 1), equating to $99.1 \%$ differential expression. Differential expression of PTPRCAP in the lymph node metastases of patients with metastatic breast cancer was statistically significant (Chart $1 ; p=1.65 \mathrm{e}-03$ ). 
To attempt to validate differential transcriptome-wide differential expression of PTPRCAP in human metastatic breast cancer, we queried a second microarray dataset ${ }^{5}$; here, we identified PTPRCAP as a differentially expressed gene in the brain metastatic tissues of patients with breast cancer (Chart 2). When sorting each of the genes expressed in brain metastases based on significance of difference as compared to primary tumors of the breast, PTPRCAP ranked 931 out of 22277 total transcripts (Chart 2), equating to $95.8 \%$ differential expression. Differential expression of PTPRCAP in the brain metastases of patients with metastatic breast cancer trended towards statistical significance (Chart $2 ; p=2.06 \mathrm{e}-02$ ). Thus, transcriptome-wide differential expression of PTPRCAP in the metastatic tissues of women with metastatic breast cancer was conserved across two independent microarray datasets, in the lymph nodes and in the brain, indicating that this may likely be a bona fide biological phenomena associated with metastatic breast cancer in humans.

PTPRCAP is expressed at higher levels in the lymph node metastases of patients with metastatic breast cancer.

We obtained exact mRNA expression levels for PTPRCAP, in primary tumors of the breast and in lymph node metastasis of patients with lymph node metastatic breast cancer to determine direction and statistical significance of change in PTPRCAP expression in lymph node metastatic tissues. We observed significantly higher expression of PTPRCAP in the lymph node metastases of patients with breast cancer as compared to primary tumors of the breast: PTPRCAP was expressed at $0.56 \pm 0.41$ units (AU) in primary tumors of the breast, while it was expressed at $1.15 \pm 0.80 \mathrm{AU}$ in lymph node metastatic tissues. The difference in PTPRCAP mRNA levels between primary tumors of the breast and lymph node metastatic tissues was statistically significant (Figure $1 ; p=0.001719$ ).

\section{PTPRCAP expression is significantly correlated with survival outcomes in human breast cancer.}

We performed Kaplan-Meier survival analysis ${ }^{8}$ in $n=889$ lymph node positive patients and $n=1309$ lymph node negative patients, to evaluate whether PTPRCAP tumor expression was correlated with survival outcomes in breast cancer. We observed a correlation between primary tumor expression of PTPRCAP and distant metastasis-free survival (DMFS) in lymph node negative patients with breast cancer, in the lower survival tertile (Figure 2). Lymph node negative patients whose primary tumors expressed low levels of PTPRCAP possessed median DMFS of 236.22 months, as compared to 222.81 months for lymph node negative patients whose tumors expressed high levels of PTPRCAP. This difference in DMFS based on PTPRCAP tumor expression in lymph node negative patients with breast cancer was statistically significant (Figure 2, Chart 3; logrank $p$-value: 0.02 ; hazard ratio: 0.74 (0.58-0.96)). Primary tumor expression of PTPRCAP was not correlated with PTPRCAP expression in lymph node positive breast cancer patients (Figure 2, Chart 3; logrank $p$-value: 0.43; hazard ratio: 1.11 $(0.85-1.46))$.

Thus, by mining published microarray data ${ }^{4,5}$ in an unbiased and systematic fashion, we identified protein tyrosine phosphatase, receptor type $\mathrm{C}$ associated protein, encoded by PTPRCAP, as among the genes whose expression was most different, transcriptome-wide, in the lymph node metastases of patients with breast cancer when compared to primary tumors of the breast; we observed significantly increased expression of PTPRCAP in lymph node metastases as compared to primary tumors of the breast. Further, we observed a correlation between PTPRCAP expression and patient survival outcomes in lymph node negative patients but not in lymph node positive patients. 


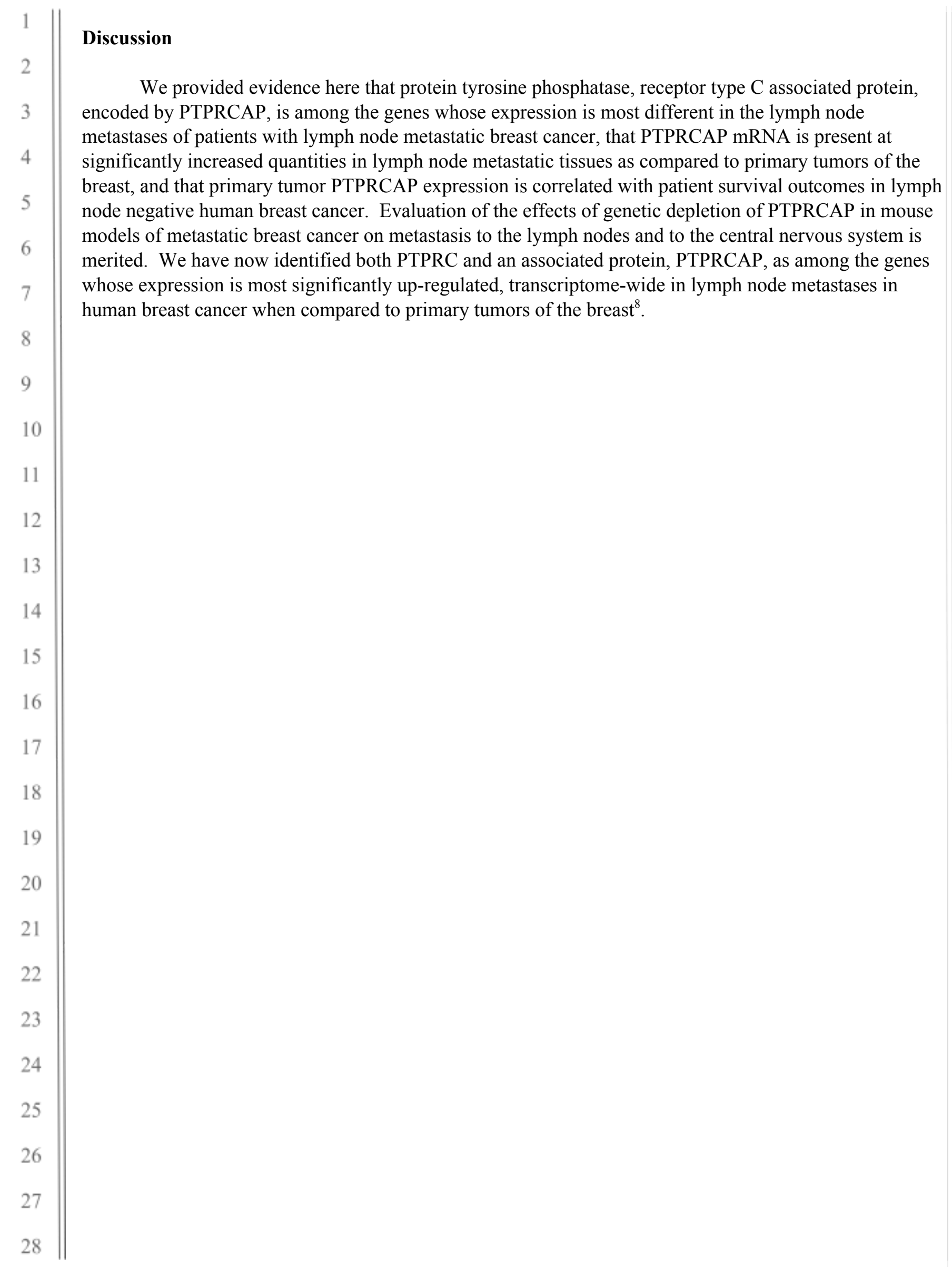




\section{References}

1. Lin, N.U., Amiri-Kordestani, L., Palmieri, D., Liewehr, D.J. and Steeg, P.S., 2013. CNS metastases in breast cancer: old challenge, new frontiers.

2. Bendell, J.C., Domchek, S.M., Burstein, H.J., Harris, L., Younger, J., Kuter, I., Bunnell, C., Rue, M., Gelman, R. and Winer, E., 2003. Central nervous system metastases in women who receive trastuzumab-based therapy for metastatic breast carcinoma. Cancer, 97(12), pp.2972-2977.

3. Tsukada, Y., Fouad, A., Pickren, J.W. and Lane, W.W., 1983. Central nervous system metastasis from breast carcinoma autopsy study. Cancer, 52(12), pp.2349-2354.

4. Weigman, V.J., Chao, H.H., Shabalin, A.A., He, X., Parker, J.S., Nordgard, S.H., Grushko, T., Huo, D., Nwachukwu, C., Nobel, A. and Kristensen, V.N., 2012. Basal-like Breast cancer DNA copy number cancer research and treatment, 133(3), pp.865-880.

5. Iwamoto, T., Niikura, N., Ogiya, R., Yasojima, H., Watanabe, K.I., Kanbayashi, C., Tsuneizumi, M., Matsui, A., Fujisawa, T., Iwasa, T. and Shien, T., 2019. Distinct gene expression profiles between primary breast cancers and brain metastases from pair-matched samples. Scientific reports, 9(1), pp.1-8.

6. Awada, A., Colomer, R., Inoue, K., Bondarenko, I., Badwe, R.A., Demetriou, G., Lee, S.C., Mehta, A.O., Kim, S.B., Bachelot, T. and Goswami, C., 2016. Neratinib plus paclitaxel vs trastuzumab plus paclitaxel in previously untreated metastatic ERBB2-positive breast cancer: the NEfERT-T randomized clinical trial. JAMA oncology, 2(12), pp.1557-1564.

7. Györffy, B., Lanczky, A., Eklund, A.C., Denkert, C., Budczies, J., Li, Q. and Szallasi, Z., 2010. An online survival analysis tool to rapidly assess the effect of 22,277 genes on breast cancer prognosis using microarray data of 1,809 patients. Breast cancer research and treatment, 123(3), pp.725-731.

8. Mamoor, S., 2021. PTPRC is differentially expressed in the lymph nodes of patients with metastatic breast cancer. 


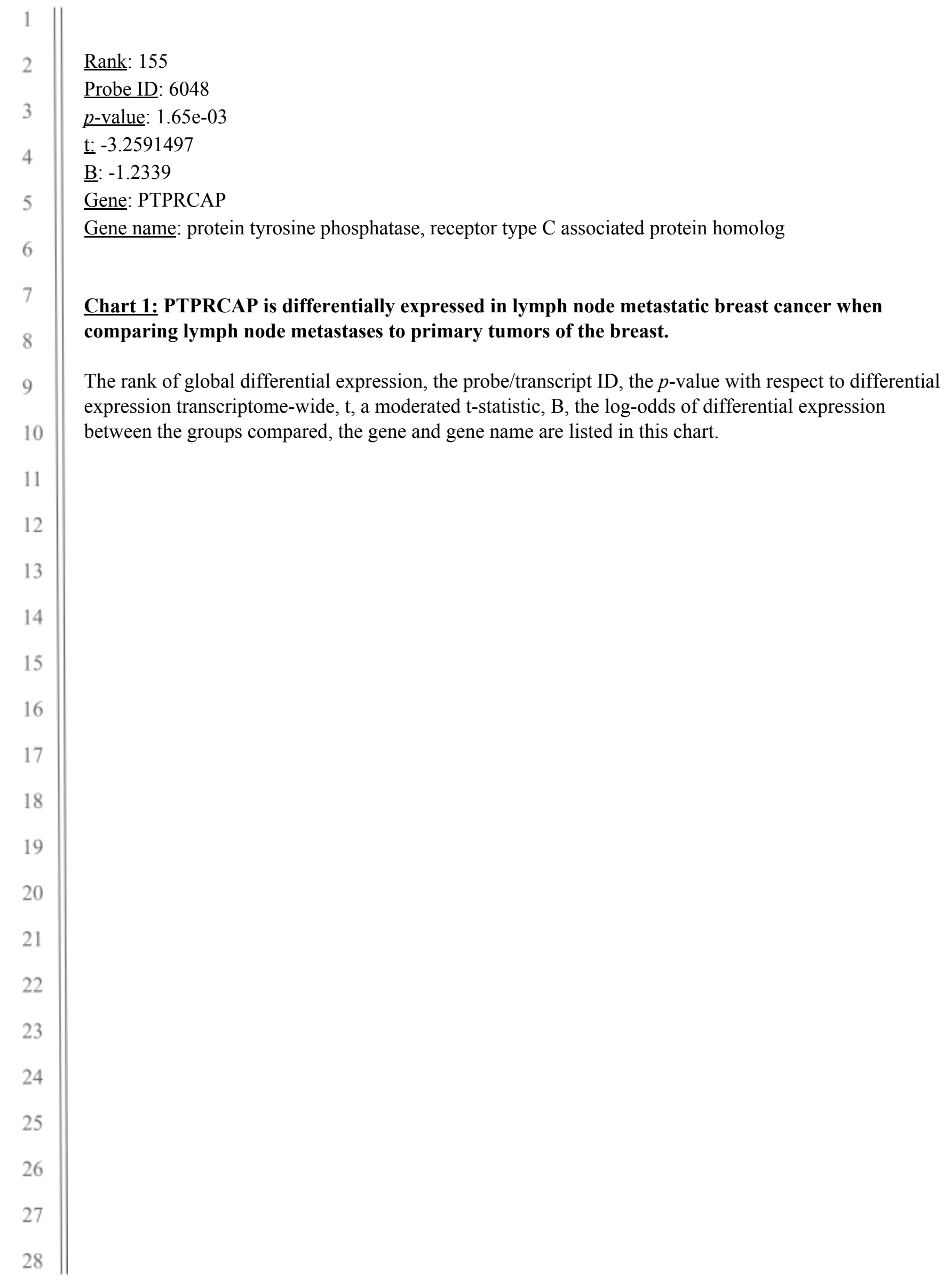




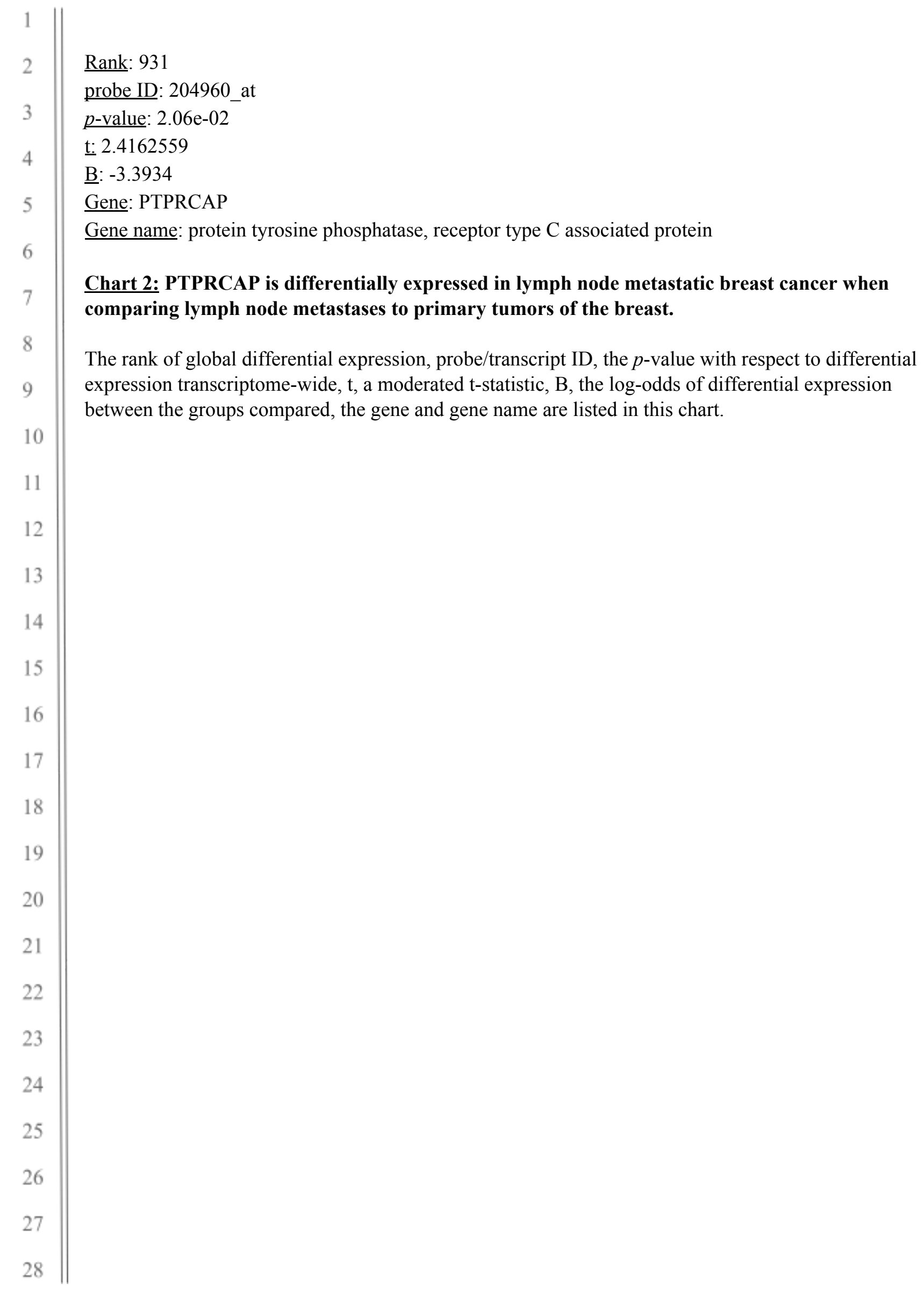




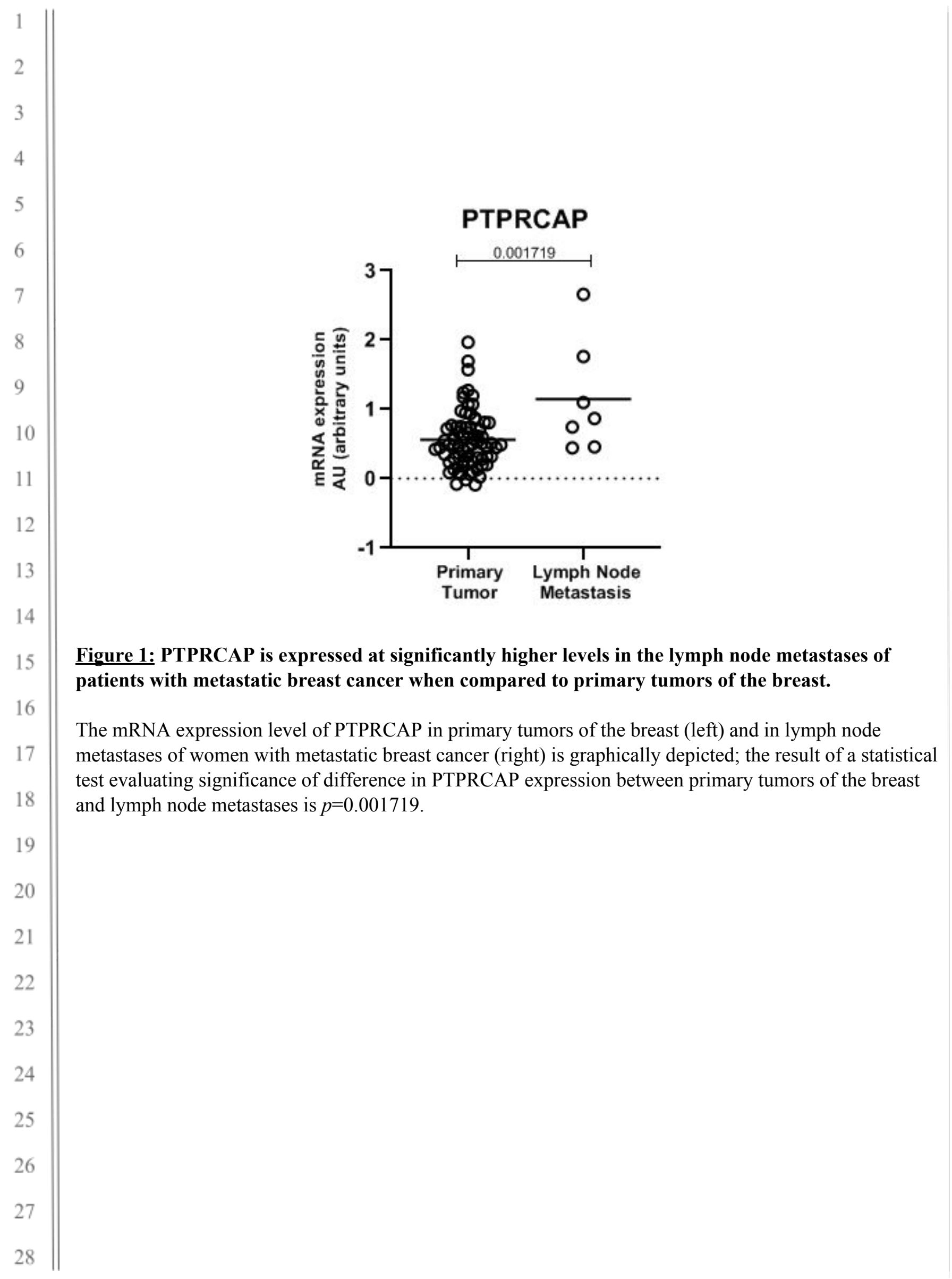



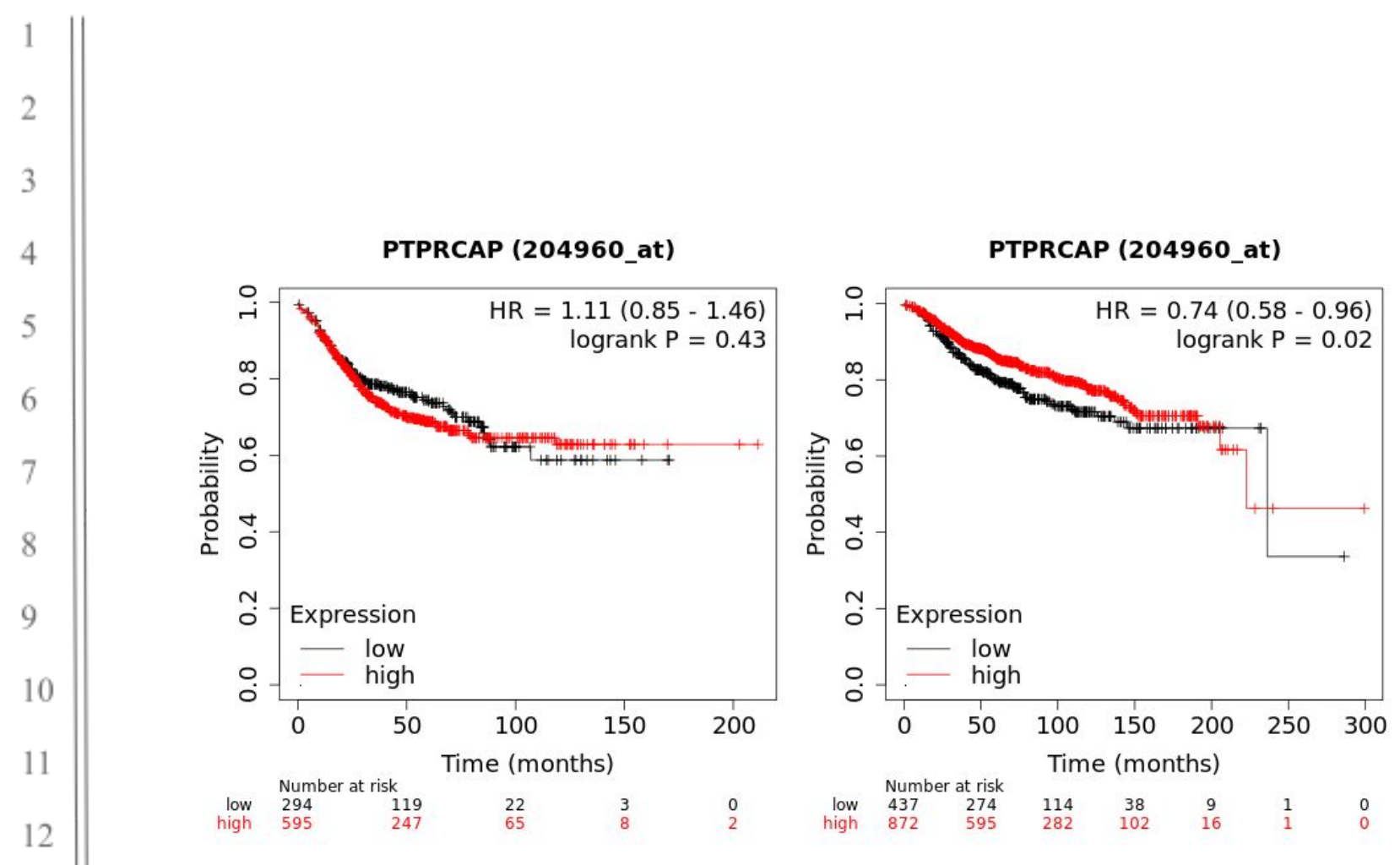

Figure 2: PTPRCAP primary tumor expression correlates with distant metastasis-free survival in patients with lymph node negative breast cancer but not in lymph node positive patients.

Depicted in these Kaplan-Meier plots is the probability of distant metastasis-free survival in $n=889$ patients with lymph node positive breast cancer (left) and $n=1309$ patients with lymph node negative breast cancer (right), stratified into two groups, based on low or high expression of PTPRCAP in patient primary tumors, in the lower survival tertile. The log rank $p$-value denoting statistical significance of difference in distant metastasis-free survival when comparing the two groups, as well as hazard ratio for this comparison is listed above. Listed below each graph is the number of patients at risk (number of patients alive) per interval, after stratification based on PTPRCAP expression; in the first interval, number at risk is number of patients alive; in each subsequent interval, number at risk is the number at risk less those who have expired or are censored. 


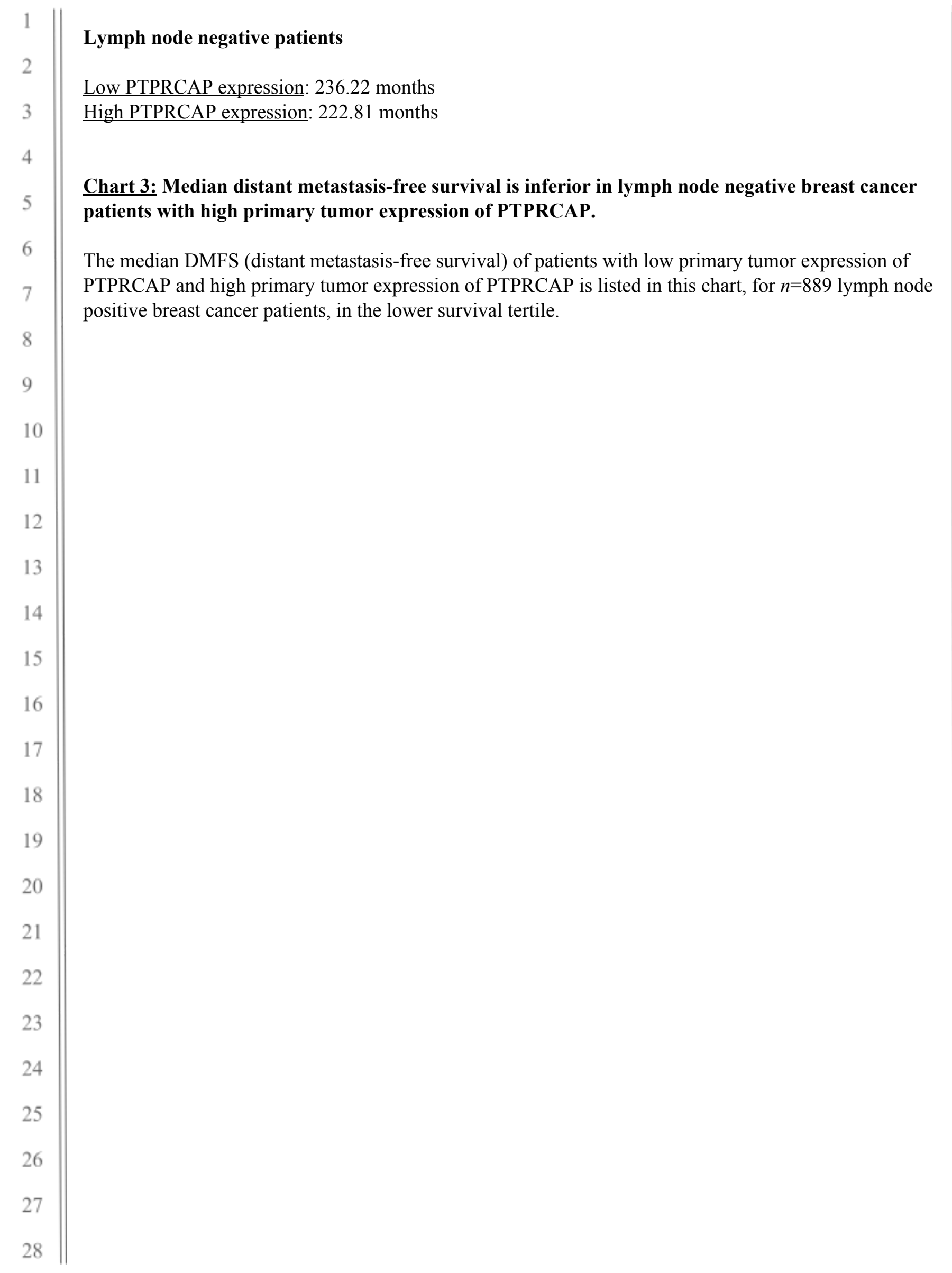

